\title{
What is a life worth living?
}

\author{
Jannick Brennum ${ }^{1}$ (D) $\cdot$ Marike Broekman ${ }^{2,3}$
}

Received: 29 September 2017 / Accepted: 7 October 2017 /Published online: 19 October 2017

(C) Springer-Verlag GmbH Austria 2017

Decompressive craniectomy (DC) has during the past decade become a treatment option for patients with life-threatening, severe, increased intracranial pressure unresponsive to nonsurgical treatments. The evidence for efficacy of DC is especially high when increased intracranial pressure is caused by malignant media infarction (MMI) [1, 2]. In this setting, DC has been proved to significantly reduce mortality. The lifesaving quality of DC might be justification enough for some to implement the procedure in routine use, but for others it needs to lead to a minimum quality of life that makes life worth living. This reflection is extremely relevant in this context because DC in MMI is very likely to save the patient's life, but it will not reverse the severe neurological deficits induced by the stroke itself. In some patients the procedure will even induce more severe neurological deficits.

The modified Rankin scale (mRS) has become the primary outcome tool in most studies of DC. When dichotomising the seven-point $\mathrm{mRS}$ into favourable and unfavourable outcomes, it was planned to set the divider between mRS3 (moderate disability - requires some help, but able to walk unassisted) and mRS4 (moderately severe disability - unable to attend to own bodily needs without assistance, and unable to walk unassisted). However, the divider was shifted and instead set

Jannick Brennum

jannick.brennum@regionh.dk

1 Department of Neurosurgery, Rigshospitalet, University of Copenhagen, Copenhagen, Denmark

2 Department of Neurosurgery, Brain Center Rudolf Magnus, University Medical Center Utrecht, Utrecht, The Netherlands

3 Department of Neurology, Massachusetts General Hospital/Harvard Medical School, Boston, MA, USA between mRS4 and mRS5 (severe disability - requires constant nursing care and attention, bedridden, incontinent) as this and not the planned dichotomisation demonstrated a significant effect of DC in MMI $[1,2]$.

Setting the cut-off mark for what life is worth living is at the centre of the article by Magnus Olivecrona from Sweden and Stephen Honeybul from Australia in the current issue of Acta Neurochirurgica [3]. They investigated what neurological outcome after DC for MMI healthcare professionals in Sweden find acceptable. The study is at the same time a replication of a similar study performed in Australia and offers a peek at potential cultural differences between Sweden and Australia [4]. These studies address the eternal question: "Yes we can, but should we?". In the current study the authors asked healthcare professionals to complete a questionnaire, where they were asked to imagine that they had just had an MMI and a DC was considered. What type of neurological deficits would they find acceptable to live with faced with the choice of accepting or declining the procedure. The participants were doctors and nurses working in intensive care, neurology or cardiology. They all received information similar to a very elaborate informed consent for the procedure, including the opportunity to ask questions, and they were introduced to a Swedish translation of the mRS [3]. In this study only $4 \%$ of the participants (24 of 609) would accept a mRS5 as outcome and $30 \%$ would accept mRS4 as outcome. When comparing with the HAMLET study [2] $75 \%$ of the patients would end up in a state that only $30 \%$ found acceptable and almost $20 \%$ would end up in a state that only $4 \%$ found acceptable.

In this editorial, we analyse the ethical aspects of DC for malignant media infarction, primarily based on the four principles often used to guide choices within the sphere of medical ethics, namely: beneficence, non-maleficence, autonomy and justice [5]. 


\section{Beneficence}

DC for MMI can save a patient's life and would thus be in line with the principle of beneficence. However, first perception is often deceivingly simple and does not cover all complexities. We believe that one would need to consider a multitude of factors in order to decide whether the DC in MMI is beneficial for the patient. One of them is obviously what the patient considers a good outcome. We believe that this decision is influenced by two elements: "when" and "where".

The most difficult issue in this context is the "when". If the responses obtained in the current study [3] are representative for the majority of patients suffering from an MMI, one question could be, would they make the same decision if they indeed had an MMI as they did when it was just a mental exercise? Would the imminent mortality change their perspective?

The next question could be - if they survived - when is the right time to evaluate their satisfaction with being alive, i.e. is life worth living. There are many examples demonstrating that we change our perspective of what is acceptable during life and we learn to live with deficits and still enjoy life even though we previously would have judged such conditions unacceptable $[6,7]$. The "when" is not restricted to being a personal "when", but can also be seen in a larger perspective as a cultural "when", where one's decision depends on the services society has to offer if you survive in a state where you are dependent on the care and aid of others - but also on the burden you impose on those close to you.

Another factor is the "where". Where do you live? Which cultural and perhaps religious setting you are brought up in and live in. In some settings, life is a priceless gift that is always worth living and in other cultural settings many will argue that they can easily imagine a quality of life poorer than being dead. We have personally recently witnessed the discussions between neurosurgeons holding these opposing positions during the ethical session at the WFNS 2017 congress in Istanbul, Turkey and at the 2017 EANS training course in Seville, Spain.

The "where" factor is apparent when comparing the two studies from Sweden and Australia, where mRS4 was an acceptable outcome for $30 \%$ of the Swedes but only for $10 \%$ of the Australians. Had the current investigation been performed in, for instance, Israel, we would expect that the difference would have been even greater.

\section{Non-maleficence or do no harm}

Even though DC for malignant stroke might be life-saving, the procedure comes at a price. Even though DC is associated with many complications that can negatively affect patients' outcomes, these complications seem quantitatively to be of minor importance. The outcome itself, often a poor mRS with patients being completely dependent on care, might be considered a negative outcome or "harm" to the patient. Performing a DC that would, on the one hand, save a patient's life, could, on the other hand, do harm to the patient, thus creating a conflict between the principles of beneficence and non-maleficence. The principle of non-maleficence would be violated when the patient who underwent DC indicates afterwards that he/she is unhappy with the outcome and would rather have died than survive with severe neurological deficits and being dependent on others. We believe the real issue here is that the patient is often not able to understand the premises of the choice to be made; the choice is often made by relatives or by the treating physician for this very reason.

As physician-assisted suicide is only legal in a small number of countries, most patients do not have the option of reversing the decision to operate for MMI. Furthermore, not all patients will have the same perception of whether their life with neurological deficits is worth living, and for the individual patient it is likely to change over time, as many will adapt and come to terms with their deficits and once again enjoy life. So one important question is how we weigh those unhappy with being alive with neurological deficits against those being dead? That is the result of performing the DC versus not performing it. Another question is how much suffering and regret of living with neurological deficits is acceptable to endure if you later come to term with your condition and once again enjoy life?

\section{Autonomy}

Olivecrona and Honeybul [3] address respect for a patient's autonomy in the light of the changing perspective over time, which may be thought of as the continuation of personhood; how do you take your future wishes into account when giving informed consent. The authors argue that such considerations are ethically problematic because they challenge the whole concept of informed consent and thereby the subject's autonomy. This may well be true. It can be seen as a challenge to the principle of autonomy, but can also be seen as a hint to the physicians that the informed consent process needs to aid the patients and relatives in a change of perspective from values ingrained in the presence towards the likely circumstances of the future with and without the procedure suggested.

\section{Justice}

Resources in healthcare are not limitless, and the principle of justice obliges us to evaluate the consequences of treatment, not for the patient, but for the rest of society. A subgroup of society will be the family of the patient. From a societal perspective, it is imperative to address the amount of resources 
spent, not only on the procedure itself but also on the care of the patient for the remainder of his or her life. If we consider the closer family perspective, we need to address the burdens and restraints survival of a close relative with severe neurological deficits impose on the family, as well as the joys and benefits of the survival of a relative will bring.

These are some of the many ethical challenges we face in the decision-making around DC for MMI. The paper by Olivecrona and Honeybul is an important contribution to the ethical debate, as it shows what some would find an acceptable outcome. However, as this study was performed in healthcare workers with assumedly similar professional, cultural, religious and societal backgrounds, it would be of importance to further explore opinions about DC in places with a different local culture and at different times.

\section{References}

1. Vahedi K, Hofmeijer J, Juettler E, Vicaut E, George B, Algra A, Amelink GJ, Schmiedeck P, Schwab S, Rothwell PM, Bousser MG, van der Worp HB, Hacke W, Decimal D, investigators H (2007) Early decompressive surgery in malignant infarction of the middle cerebral artery: a pooled analysis of three randomised controlled trials. Lancet Neurol 6:215-222

2. Hofmeijer J, Kappelle LJ, Algra A, Amelink GJ, van Gijn J, van der Worp HB, investigators H (2009) Surgical decompression for spaceoccupying cerebral infarction (the Hemicraniectomy After Middle cerebral artery infarction with Life-threatening Edema Trial [HAMLET]): a multicentre, open, randomised trial. Lancet Neurol $8: 326-333$

3. Olivecrona M, Honeybul S (2017) Study of Swedish health care personnel's regarding acceptable outcome following decompressive hemicraniectomy for ischemic stroke. Acta Neurochir xxx:yyyy-zzzz

4. Honeybul S, Ho KM, Blacker DW (2016) ORACLE stroke study: opinion regarding acceptable outcome following decompressive hemicraniectomy for ischemic stroke. Neurosurgery 79:231-236

5. Beauchamp TL, Childress JF (2009) Principles of biomedical ethics, 6th edn. Oxford University Press, New York

6. Brennum J, Maier CM, Almdal K, Engelmann CM, Gjerris M (2015) Primo non nocere or maximum survival in grade 2 gliomas? A medical ethical question. Acta Neurochir 157(5):811-812

7. Honeybul S, Gillet GR, KM HO, Janzen C, Kruger K (2015) Longterm survival with unfavourable outcome: a qualitative and ethical analysis. J Med Ethics 41(12):963-969 